\title{
Protected $\mathbf{0}-\pi$ states in SIsFS junctions for Josephson memory and logic
}

S. V. Bakurskiy, N. V. Klenov, I. I. Soloviev, N. G. Pugach, M. Yu. Kupriyanov, and A. A. Golubov

Citation: Appl. Phys. Lett. 113, 082602 (2018); doi: 10.1063/1.5045490

View online: https://doi.org/10.1063/1.5045490

View Table of Contents: http://aip.scitation.org/toc/apl/113/8

Published by the American Institute of Physics

\section{Articles you may be interested in}

A distributed electrical model for superconducting nanowire single photon detectors

Applied Physics Letters 113, 082601 (2018); 10.1063/1.5040150

Electromagnetic proximity effect in planar superconductor-ferromagnet structures

Applied Physics Letters 113, 022601 (2018); 10.1063/1.5037074

Superconducting nano Josephson junctions patterned with a focused helium ion beam

Applied Physics Letters 113, 022604 (2018); 10.1063/1.5042105

Increased multiplexing of superconducting microresonator arrays by post-characterization adaptation of the onchip capacitors

Applied Physics Letters 113, 082603 (2018); 10.1063/1.5040968

Spin-current coherence peak in superconductor/magnet junctions

Applied Physics Letters 112, 232601 (2018); 10.1063/1.5027456

AC magnetic field sensing using continuous-wave optically detected magnetic resonance of nitrogen-vacancy centers in diamond

Applied Physics Letters 113, 082405 (2018); 10.1063/1.5024401

\section{Conference Proceedings}




\title{
Protected 0- $\pi$ states in SIsFS junctions for Josephson memory and logic
}

\author{
S. V. Bakurskiy, ${ }^{1,2}$ N. V. Klenov, ${ }^{1,2,3,4}$ I. I. Soloviev, ${ }^{1,2}$ N. G. Pugach, ${ }^{1,5}$ M. Yu. Kupriyanov, ${ }^{1,2}$ \\ and A. A. Golubov $2,6, a)$ \\ ${ }^{1}$ Skobeltsyn Institute of Nuclear Physics, Lomonosov Moscow State University 1(2), Leninskie Gory, \\ Moscow 119234, Russian Federation \\ ${ }^{2}$ Moscow Institute of Physics and Technology, Dolgoprudny, Moscow Region 141700, Russian Federation \\ ${ }^{3}$ Faculty of Physics, M.V. Lomonosov Moscow State University, Leninskie Gory, 119992 Moscow, Russia \\ ${ }^{4}$ All-Russian Research Institute of Automatics n.a. N.L. Dukhov (VNIIA), 127055 Moscow, Russia \\ ${ }^{5}$ National Research University Higher School of Economics, 101000 Moscow, Russia \\ ${ }^{6}$ Faculty of Science and Technology and MESA+ Institute for Nanotechnology, University of Twente, \\ 7500 AE Enschede, The Netherlands
}

(Received 20 June 2018; accepted 3 August 2018; published online 21 August 2018)

\begin{abstract}
We study the peculiarities in current-phase relations (CPR) of the SIsFS junction in the region of 0 to $\pi$ transition. These CPR consist of two independent branches corresponding to 0 - and $\pi$-states of the contact. We have found that depending on the transparency of the SIs tunnel barrier, the decrease in the s-layer thickness leads to transformation of the CPR shape going in the two possible ways: either one of the branches exists only in discrete intervals of the phase difference $\varphi$ or both branches are sinusoidal but differ in the magnitude of their critical currents. We demonstrate that the difference can be as large as $10 \%$ under maintaining superconductivity in the s layer. An applicability of these phenomena for memory and logic application is discussed. Published by AIP Publishing.

https://doi.org/10.1063/1.5045490
\end{abstract}

Josephson junctions with ferromagnetic $(F)$ layers in the weak link region are considered as promising control elements in a superconducting memory compatible with RSFQ logic circuits. ${ }^{1-6}$ The presence of two or more ferromagnetic layers in the weak-coupling region makes it possible to control the magnitude of the critical current $J_{C}$ of these junctions by changing the mutual orientation of $F$ film magnetization vectors. ${ }^{7-13}$ It is necessary to mention that the large number of ferromagnetic layers in the weak-coupling area is accompanied by degradation of $J_{C}$ by virtue of the larger number of interfaces in the structure and owing to the strong suppression of superconducting correlations in each of the F layers.

In Refs. 14-19, it was shown that the required changes in $J_{C}$ can also be ensured in SFS or SIsFS structures with a single ferromagnetic layer. The remagnetization of the ferromagnetic layer in these junctions shifts the position of the maximum in Fraunhofer-like dependence of $J_{C}$ on the external magnetic field resulting in a change of $J_{C}$ magnitude at zero field. This principle was extended in magnetic rotary valves $^{20,21}$ where the switching effect in $J_{C}$ magnitude was achieved by changing the direction of the inplane $F$ film magnetization.

It should be noted that magnetization reversal processes significantly increase the characteristic response time of the SFS control memory elements in comparison with the characteristic switching time of Josephson contacts in single flux quantum (SFQ) logic circuits. In order to overcome this drawback, it was suggested in Ref. 22 to use SIs-F/N-S contacts, where a thin s-layer can be subdivided on superconducting domains with a phase shift of $\pi$. However, the implementation of the above mentioned proposals is a rather complicated technological task.

\footnotetext{
a) Author to whom correspondence should be addressed: a.golubov@ utwente.nl
}

The promising concept of the Josephson memory with electrical control can be also realized using the phenomenon of the coexistence of the two metastable states in the vicinity of $0-\pi$ transition. $^{23,24}$ For instance, these states can be achieved inside the region of the $0-\pi$ transition of the junction with the ferromagnetic layer ${ }^{25-28}$ or in the junctions with two noncollinearly magnetized hard ferromagnets. ${ }^{29,30}$ The conditions for the existence of metastable states essentially depend both on the material parameters of the contacts and on their geometry.

The purpose of this article is to propose the concept of the control element for memory based on our finding of noticeable difference between critical current in 0 - and $\pi$-states in a certain range of the s-layer thickness $d_{s}$ in SIsFS junctions.

Figure 1 schematically shows the principle of operation of the SIsFS structure in comparison with SFS or S-F/N-S devices. This figure demonstrates the evolution of the current-phase relation (CPR) $J_{S}(\varphi)$ and energy-phase relation (EPR) $E_{S}(\varphi)$ of SFS or S-F/N-S junctions and SIsFS structures in a vicinity of 0 to $\pi$ transition, which occurs with the increase in $\mathrm{F}$ layer thickness $d_{F}$.

For the SFS junction, the amplitude of the second harmonic in CPR is positive $27,28,31,32$ at any transition point. The $0-\pi$ transition is going through the region of coexistence of the states with 0 and $\pi$ phase differences [see Fig. 1(a)]. During the transition, the depths of the corresponding minima in the $E_{S}(\varphi)$ relation are changing continuously until one of them disappears.

In the Josephson junctions with parallel 0 - and $\pi$-channels for the supercurrent flow inside a weak link region, ${ }^{33-37}$ e.g., S-F/N-S contacts, the amplitudes of the first harmonic in CPR in the channels have opposite signs and compensate each other. At the same time, the amplitudes of the second harmonic have negative signs in both channels. In this situation, the transition from the initial 0 state to the final $\pi$ state 

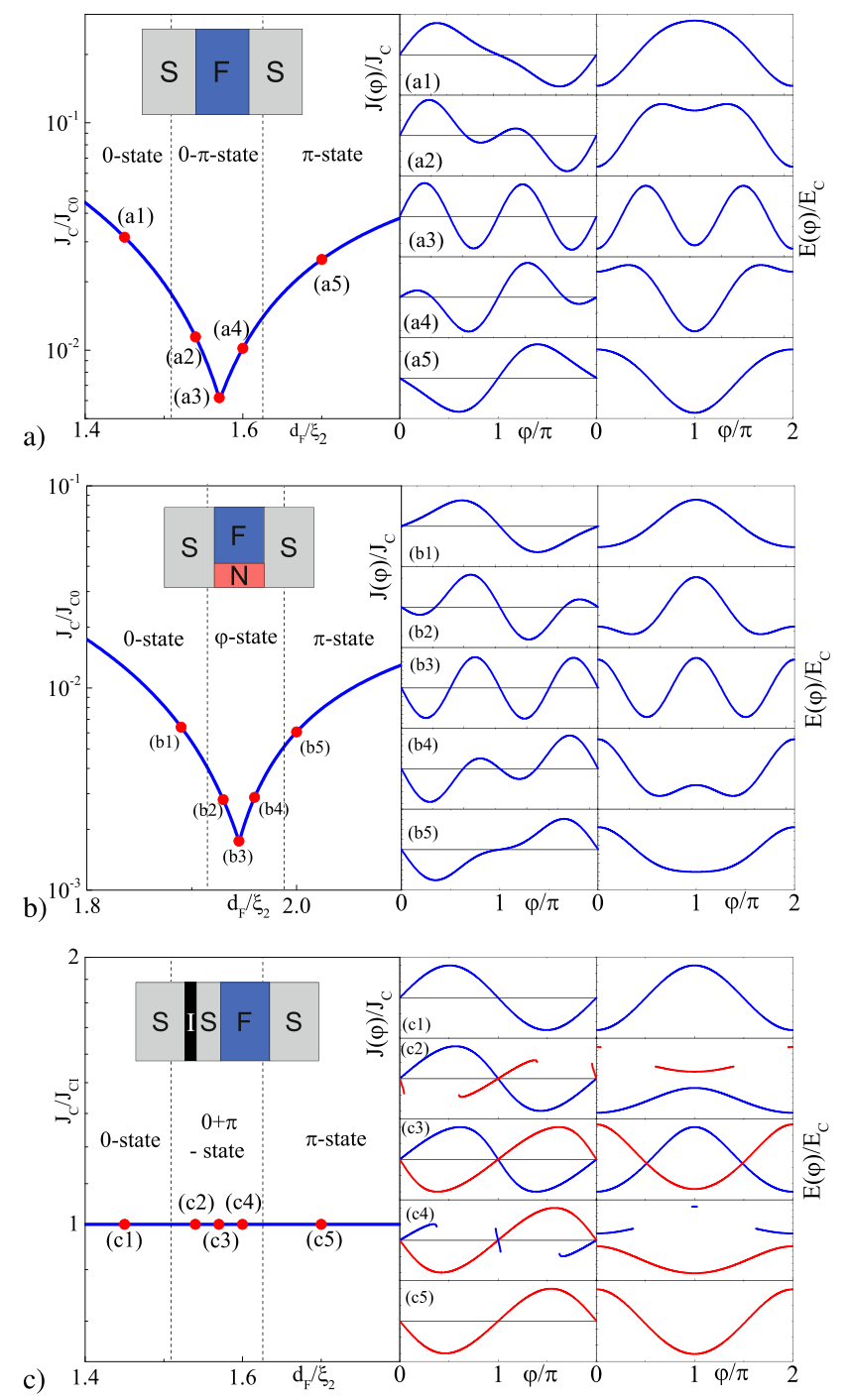

FIG. 1. Typical behaviour of (a) SFS, (b) S-F/N-S, and (c) SIsFS junction characteristics in a vicinity of $0-\pi$ transition. Each block (a)-(c) includes 3 parts. The left one demonstrates the dependence of the critical current $J_{C}$ versus the ferromagnet layer thickness $d_{F}$. The middle row contains CPRs of the junctions at the certain $d_{F}$ marked by the red dots (1)-(5) on the $J_{C}\left(d_{F}\right)$ dependence. Finally, the right row demonstrates EPRs at the same points.

takes place via formation of the so-called $\varphi$-state. With the increase in $d_{F}$ [see Fig. 1(b)], the minimum in $E_{S}(\varphi)$ located at $\varphi=0$ splits into two minima located at some, $\pm \varphi\left(d_{F}\right)$, and this $\varphi\left(d_{F}\right)$ tends to $\varphi=\pi$ at the end of the transition.

In both $0-\pi$ transitions presented in Figs. 1(a) and 1(b), the $J_{C}\left(d_{F}\right)$ curves are $\mathrm{V}$-shaped with a strong suppression of the critical current during the transitions. The metastable states in both structures generally have different barriers, which would be exceeded to switch device into resistive regime. It provides opportunity to read the state, although after that the state will be erased.

Contrary to that, in SIsFS devices, ${ }^{38-42}$ it is possible to realize the mode of operation in which magnitude of $J_{C}$ of SIs part of the structure is smaller than the amplitude of the second harmonic in CPR of its sFS part. In this case [see Fig. 1 (c) $],\left|J_{C}\right|$ is constant during the $0-\pi$ transition, ${ }^{40,42}$ although the current-phase relations undergo significant transformations and become multivalued. For relatively large layer thickness $d_{s}$, there is the domain of SIsFS junction parameters providing its stay either in the 0 - or in $\pi$-ground state. The critical current is determined by SIs part of the junction and is exactly the same for both the states. In this domain, a transition from one ground state to another is not possible by a continuous adiabatic variation of the phase $\varphi$ and the states can be used for storing information since the energy barrier that separated them is higher than the energy of the tunnel junction, thus protecting the system against accidental switching.

In order to model the SIsFS structure, we suppose that the condition of a dirty limit is fulfilled for all metals and that effective electron-phonon coupling constant is zero in the F layer. Under the above conditions, the problem can be analyzed in the framework of the Usadel equations ${ }^{43}$ with Kupriyanov-Lukichev boundary conditions ${ }^{44}$ at the interfaces. The boundary-value problem was solved numerically using the algorithm developed in Ref. 42. For simplicity, we assume below that the resistivities $\rho$ and coherence lengths $\xi=\left(D / 2 \pi T_{C}\right)^{1 / 2}$ of the SIsFS junction materials are the same. Here, $T_{C}$ is a critical temperature and $D$ is a diffusion coefficient of the superconducting material.

Based on our previous investigations, ${ }^{38,42}$ we have fixed the set of SIsFS junction parameters that ensure an occurrence of SIsFS contact in the vicinity of the $0-\pi$ transition at large s layer thickness $d_{s}=5 \xi: d_{F}=0.46 \xi, T=0.26 T_{C}$, exchange energy $H=10 \pi T_{C}$, suppression parameters of SF interface $\gamma_{B S F}=R_{B S F} \mathcal{A} / \xi \rho=0.3$, and SIs interface $\gamma_{B I}=R_{B I} \mathcal{A} / \xi \rho$ $=5000$. Here, $R_{B}$ and $\mathcal{A}$ are the resistance and area of the corresponding interface. With this choice of parameters, the weakest link of the SIsFS structure is located at the tunnel barrier, thus providing the coexistence of the two independent CPR branches [see example in the panel (c3) in Fig. 1(c)].

We start with the calculation of the dependence of $J_{C}$ magnitude on $d_{s}$, shown as a blue line in Fig. 2. It has a common form with a rapid drop of the critical current near the critical thickness $d_{s C} \approx 2.7 \xi$. There are two independent processes going in the vicinity of this point. The first one is a shifting of the position and narrowing of the width of the $0-\pi$ transition during the decrease in the $d_{s}$, due to the change in the effecting boundary conditions on the $\mathrm{F}$ layer. ${ }^{16}$

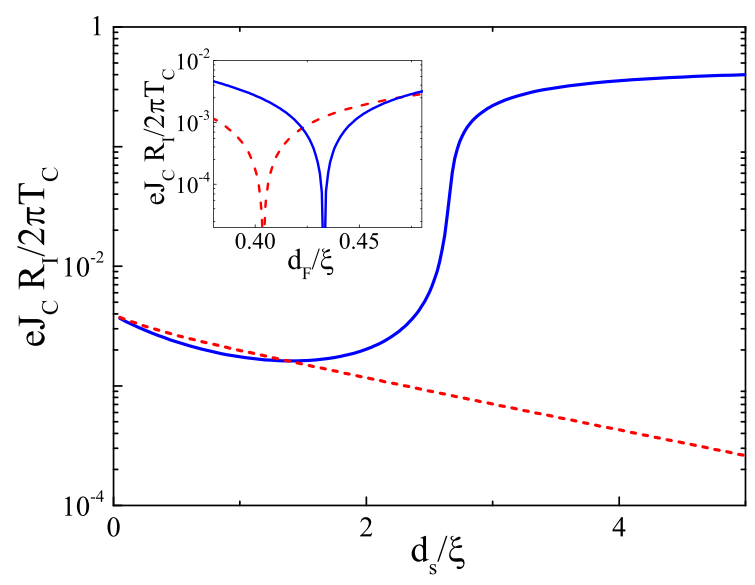

FIG. 2. Magnitude of the critical current $J_{C}$ of the SIsFS (solid blue) and the SInFS (dashed red) junctions versus thickness of the middle layer $d_{s}$ calculated in the vicinity of $0-\pi$ transition at $d_{F}=0.46 \xi$ and $T=0.26 T_{C}$. Inset: Critical current $J_{C}$ of the SIsFS (solid blue) and the SInFS (dashed red) junctions versus thickness of the F-layer $d_{F}$ for s-layer thickness $d_{s}=1 \xi$ much smaller than $d_{s C}$. 
The inset in Fig. 2 shows that transformation significantly differs from the process studied in the SInFS junction, ${ }^{45}$ since the residual pairing locks the phases of different Matsubara frequencies. This phenomenon leads to collapse of the 0-branch of the CPR at the $d_{s}$ near the critical point.

The second phenomenon is the deviation of the pair amplitude in the thin s-electrodes for 0 and $\pi$ states due to the different symmetry of the anomalous Green functions predicted and found experimentally in the sFS junctions with thin s-electrodes. ${ }^{46-48}$ This effect can modify the critical currents of the tunnel SIs junction due to the change in the s-layer properties in 0 and $\pi$ states.

The relative impact of these processes on the SIsFS structure properties depends on the tunnel suppression parameter $\gamma_{B I}$. First, we consider the system at $\gamma_{B I}=5000$, when the collapse of the 0-branch occurs.

Figure 3(a) demonstrates the evolution of the shape of $J_{S}(\varphi)$ dependence with the decrease in the s layer thickness. It is seen that the magnitudes of critical current of both the CPR branches are monotonically decreasing, while the shape of the curves transforms in different ways. The CPR of the $\pi$ ground state remains sinusoidal at any $d_{s}$ during the $d_{s}$ decrease. The CPR of the 0-branch at some $d_{S}$ becomes broken and disappears in the significant interval of phases. At $d_{s} \lesssim 2.7 \xi$, it completely disappears and only the $\pi$ ground

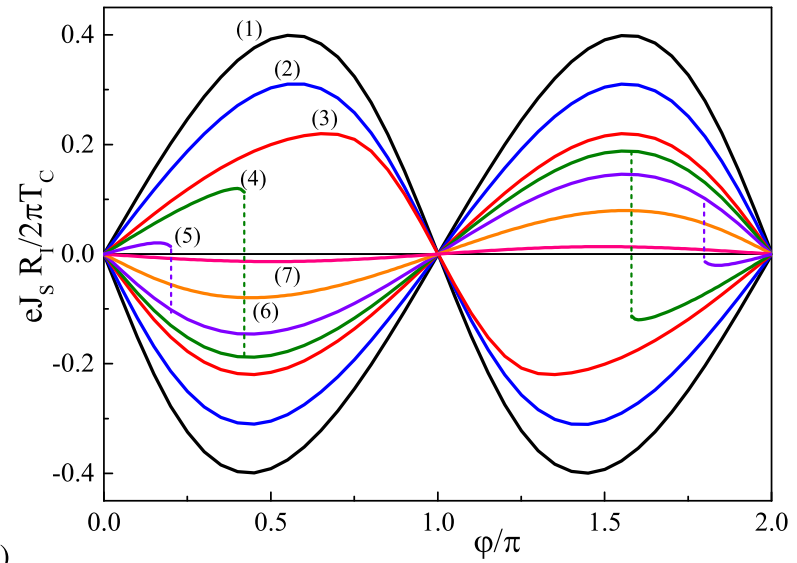

a)

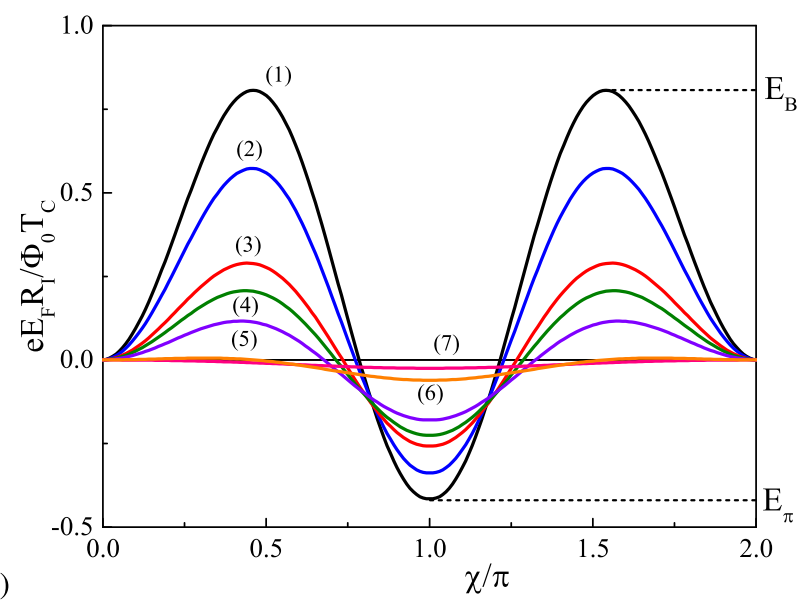

FIG. 3. The current-phase relation $J_{S}(\varphi)$ of the SIsFS junction (a) and the energy-phase relation $E_{F}(\chi)$ of the corresponding sFS junction (b) for the set of the different thicknesses of the s-layer $d_{s}$ : (1) $5 \xi$, (2) $3.5 \xi$, (3) $3 \xi$, (4) $2.9 \xi$, (5) $2.8 \xi$, (6) $2.7 \xi$, and (7) $2.6 \xi$. CPRs include 0 and $\pi$ branches in the cases (1)-(5) and only $\pi$ branch for (6) and (7). The calculations were done in the vicinity of $0-\pi$ transition at $d_{F}=0.46 \xi, T=0.26 T_{C}$ and $\gamma_{B}=5000$. state still exists. At the smaller $d_{s} \leqslant 2.5 \xi$, critical current of the $\pi$-branch is strongly suppressed due to intensive suppression of the superconductivity in the s-layer by the inverse proximity effect.

To understand the physics behind the $J_{S}(\varphi)$ transformations shown above, it is convenient to use the so-called lump junction model ${ }^{42}$ and consider the SIsFS junction as a series connection of SIs and sFS contacts with finite thickness $d_{s}$ of the s electrode. The self-consistent problem for the sFS junction was solved numerically with free boundary condition $d \Phi_{s} / d x=0$ at the Is interface. The choice of the initial pair potential in the iterative self-consistent calculation permits us to find the magnitudes of Usadel functions $\Phi_{s}$ at the Is interface for both 0 and $\pi$-states. Taking into account that the supercurrent flowing across the SIsFS structure is essentially small compared to $\mathrm{S}$ and $\mathrm{S}$ films' depairing current and that the $d_{s}$ thicknesses of interest exceed $2.5 \xi$, we can neglect a dependence of $\Phi_{s}$ magnitudes on phase differences $\chi$ and $\chi_{1}$ across both sFS and SIs junctions, respectively. Under this assumption, the critical current, $I_{C}$, and energy phase relation, $E_{I}\left(\chi_{1}\right)$, of SIs contact can be calculated using standard well-known expressions

$$
\begin{aligned}
& \frac{e I_{c} R_{I}}{2 \pi T_{C}}=\frac{T}{T_{C}} \operatorname{Re} \sum_{\omega>0} \frac{\Delta_{0} \Phi_{s}}{\sqrt{\left(\omega^{2}+\Delta_{0}^{2}\right)\left(\omega^{2}+\Phi_{s}^{2}\right)}}, \\
& E_{I}\left(\chi_{1}\right)=E_{C I}\left(1-\cos \left(\chi_{1}\right)\right), \quad E_{C I}=\frac{\Phi_{0} I_{c}}{2 \pi},
\end{aligned}
$$

where $R_{I}$ is the resistance of the tunnel barrier and $\Phi_{0}$ is the magnetic flux quantum.

In the considered approximation, the same quantities $\Phi_{s}$ can be used as boundary conditions of the first kind in calculating $\mathrm{CPR} J_{S}(\chi)$ and EPR

$$
E_{F}(\chi)=\frac{\Phi_{0}}{2 \pi} \int_{0}^{\chi} J_{S}(\mu) d \mu
$$

of sFS contact [see Fig. 3(b)].

Figure 3(b) demonstrates that the $E_{F}(\chi)$ dependence has the double-well form with the two minima at $\chi=\pi$ and $\chi$ $=0$ separated by a potential barrier $E_{B}$. The decrease in $d_{s}$ is accompanied by the suppression of the barrier height, $E_{B}\left(d_{s}\right)$. At $d_{s} \leqslant 2.7 \xi$, the potential barrier completely disappears and the sFS contact stays only in the $\pi$ ground state. The depth of the potential well for the $\pi$-state $E_{\pi}$ also decreases rapidly with a further decrease in $d_{s}$.

We summarize the dependence of the characteristical energies $E_{B}$ (dashed black), $E_{B}-E_{\pi}$ (dash-dotted green), and $E_{C I}$ versus thickness $d_{s}$ in Fig. 4. The energy of tunnel junction $E_{C I}$ is calculated in the frame of the lumped junction model independently for 0 - (solid red) and $\pi$ - (short-dashed blue) states of the sFS-electrode. It is seen that they practically coincide with each other for $d_{s}>2.8 \xi$. The blue dots mark the critical energies of the SIsFS junction shown in Fig. 3. They correlate well with the results obtained in the lumped junction model, thus demonstrating the accuracy of our approach.

It follows from Fig. 4 that in the wide range of $d_{s}$, the tunnel energy $E_{C I} \ll E_{B}, E_{B}-E_{\pi}$. These conditions provide 


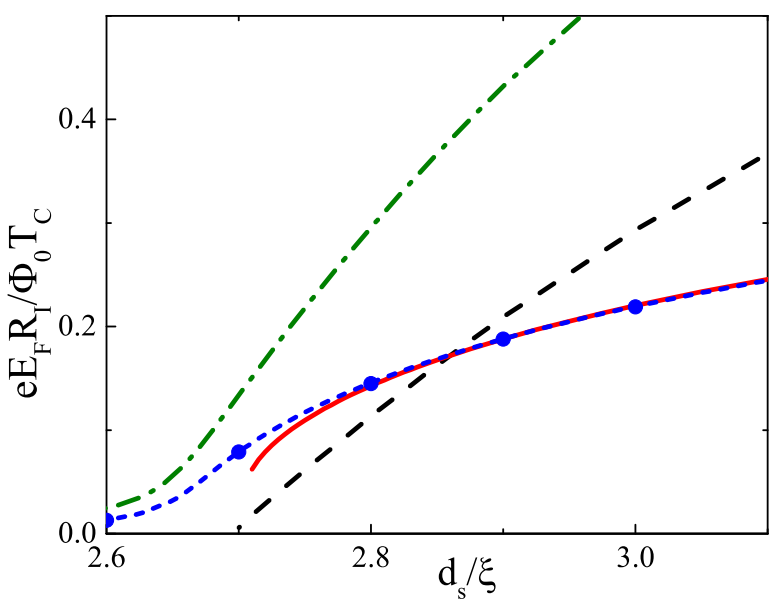

FIG. 4. The characteristical energies of the SIs and sFS junctions: barrier heights in $\mathrm{sFS}$ junction $E_{B}$ (dashed black) and $E_{B}-E_{\pi}$ (dash-dotted green) and the energy of SIs junction $E_{C I}$ in the 0 -(solid red) and $\pi$-(short-dashed blue) states versus the thickness of s layer $d_{s}$. The blue dots mark the energies calculated from the CPR of SIsFS junction demonstrated in Fig. 3(a).

the existence of two independent continuous branches of CPR in the SIsFS junction for both the 0 - and $\pi$-states as it is seen in Fig. 3(a). With the decrease in $d_{s}$, the barrier height $E_{B}$ is suppressed more rapidly than $E_{C l}$. At $d_{s} \approx 2.8 \xi$, the energies $E_{B}$ and $E_{C I}$ become comparable and the adiabatic increase in $\varphi$ leads to the escape of the system from the metastable to the ground state resulting in a discontinues jump in $J_{S}(\varphi)$. Finally, at the critical thickness $d_{s} \approx 2.7 \xi$, the barrier completely vanishes, leading to the disappearance of the 0 -branch. At the same time, the barrier for the $\pi$-state significantly exceeds $E_{C I}$ in the whole considered interval.

In the region near the critical thickness $d_{s} \approx 2.7 \xi$, the energies of the tunnel SIs junction corresponding to 0 -(red) and $\pi$-(blue) states deviate. The absolute values of Green function $\Phi_{s}$ and pair potential $\Delta$ on the tunnel SIs interface are different for 0 and $\pi$ states. In contrast to Refs. 46-48, we find that the $\pi$-state has a larger value of $\Delta$ and larger critical current.

The difference between 0 and $\pi$-states provides a significant influence on the CPR and critical current of the SIsFS structure at the larger values of tunnel layer parameter $\gamma_{B I}$. The increase in $\gamma_{B I}$ does not modify the barrier height $E_{B}\left(d_{s}\right)$ of the sFS-junction but proportionally decreases the $E_{C I}\left(d_{s}\right)$, shifting the cross-point between them to the critical thickness $d_{s C}$.

For instance, for the value of suppression parameter $\gamma_{B I}$ $=5 \times 10^{7}$, direct calculations of the SIsFS structure show that both the branches of CPR have a sinusoidal shape and are defined for all phases $\varphi$ even at $d_{s}=2.72 \xi$ (see Fig. 5). It is important to note that for this particular case, the critical currents $J_{C 0}$ and $J_{C \pi}$ of 0 - and $\pi$-CPR branches are different from each other and this difference $\left(J_{C \pi}-J_{C 0}\right) / J_{C 0}$ is of the order of $10 \%$.

The ability of the structure to be in one of the two states differing in their critical currents can find applications in superconductor logic and memory devices. Information on the state of the SIsFS structure can be obtained by setting a current pulse, with an amplitude, $J$ in the range $J_{C 0}<J<J_{C \pi}$. It is determined by the absence $(\pi$-state) or by the generation

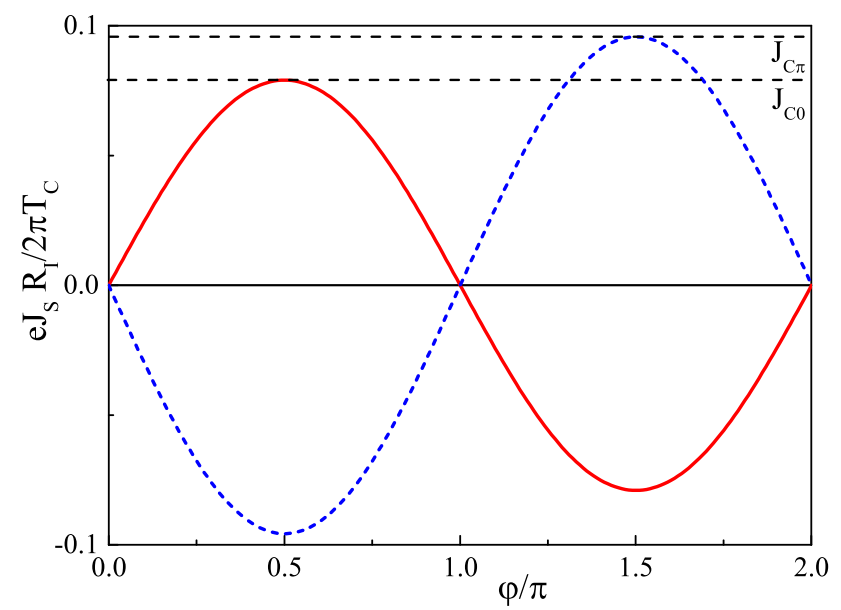

FIG. 5. (a) The 0-(solid red) and $\pi$-(dashed blue) branches of the CPR $J_{S}(\varphi)$ of the SIsFS junction with thin s-layer $d_{s}=2.72 \xi$ and strong $\gamma_{B}=5 \times 10^{7}$.

(0-state) of the corresponding voltage pulse. Reading the state of the element will be non-destructive, if the incoming energy is insufficient to open the channel of tunneling through the energy barrier separating the 0 - and $\pi$-states. The recording is possible with current pulses, which have an amplitude exceeding the critical current of the sFS junction and switch the system from the 0 to $\pi$-state and back. ${ }^{24}$

The drawback of this concept is in strong limitation on the thickness of both the superconducting and ferromagnetic layers and the smallness of the critical currents. The thickness of the s layer should be close to its critical value with an accuracy of the order of $0.1 \xi \approx 1 \mathrm{~nm}$, while the thickness of the ferromagnet should ensure the occurrence of that $0-\pi$ transition. On the other hand, recent experiments ${ }^{27,28}$ have demonstrated the feasibility of this task.

The advantages of the proposed SIsFS element compared to spin-valves ${ }^{10-13}$ or single flux quantum (SFQ) devices $^{49,50}$ are obvious. The proposed control memory element stores information only in the phase difference across the junction in the steady state. Thus, it makes use of its own intrinsic properties to store information, so that no remagnetization of the F layers or holding a flux quantum is needed.

In this sense, the bistable SIsFS device may serve as a truly Josephson memory device, which permits the reduction of size of the auxiliary circuits or even provide an alternative to the SFQ concept of superconducting electronics.

The authors acknowledge helpful discussion with V. V. Ryazanov and V. V. Bol'ginov. The design of the memory concept was supported by the Russian Science Foundation (12-17-01079) and numerical study of the sFS junction was done with support of RFBR (18-32-00672 mol-a).

${ }^{1}$ M. Eschrig, Rep. Prog. Phys. 78, 104501 (2015).

${ }^{2}$ J. Linder and J. W. A. Robinson, Nat. Phys. 11, 307 (2015).

${ }^{3}$ M. G. Blamire and J. W. A. Robinson, J. Phys. Condens. Matter 26, 453201 (2014).

${ }^{4}$ J. W. Lu, E. Chen, M. Kabir, V. R. Stan, and S. A. Wolf, Int. Mater. Rev. 61, 456 (2016).

${ }^{5}$ O. A. Mukhanov, "Digital processing, superconductor digital electronics," in Applied Superconductivity: Hand-Book on Devices and Applications, edited by P. Seidel (Wiley-VCH Verlag GmbH \& Co. KGaA, Weinheim, Germany, 2015). 
${ }^{6}$ I. I. Soloviev, N. V. Klenov, S. V. Bakurskiy, M. Yu Kupriyanov, A. L. Gudkov, and A. S. Sidorenko, Beilstein J. Nanotechnol. 8, 2689 (2017).

${ }^{7}$ A. A. Golubov, M. Y. Kupriyanov, and E. Il'ichev, Rev. Mod. Phys. 76, 411 (2004).

${ }^{8}$ A. I. Buzdin, Rev. Mod. Phys. 77, 935 (2005).

${ }^{9}$ F. S. Bergeret, A. F. Volkov, and K. B. Efetov, Rev. Mod. Phys. 77, 1321 (2005).

${ }^{10}$ C. Bell, G. Burnell, C. W. Leung, E. J. Tarte, D.-J. Kang, and M. G. Blamire, Appl. Phys. Lett. 84, 1153 (2004).

${ }^{11}$ M. A. E. Qader, R. K. Singh, S. N. Galvin, L. Yu, J. M. Rowell, and N. Newman, Appl. Phys. Lett. 104, 022602 (2014).

${ }^{12}$ B. Baek, W. H. Rippard, S. P. Benz, S. E. Russek, and P. D. Dresselhaus, Nat. Commun. 5, 3888 (2014).

${ }^{13}$ B. M. Niedzielski, T. J. Bertus, J. A. Glick, R. Loloee, W. P. Pratt, Jr., and N. O. Birge, Phys. Rev. B 97, 024517 (2018).

${ }^{14}$ V. V. Bol'ginov, V. S. Stolyarov, D. S. Sobanin, A. L. Karpovich, and V. V. Ryazanov, Pis'ma v ZhETF 95, 408 (2012) [JETP Lett. 95, 366 (2012)].

${ }^{15}$ T. I. Larkin, V. V. Bol'ginov, V. S. Stolyarov, V. V. Ryazanov, I. V. Vernik, S. K. Tolpygo, and O. A. Mukhanov, Appl. Phys. Lett. 100, 222601 (2012).

${ }^{16}$ I. V. Vernik, V. V. Bol'ginov, S. V. Bakurskiy, A. A. Golubov, M. Yu. Kupriyanov, V. V. Ryazanov, and O. A. Mukhanov, IEEE Trans. Appl. Supercond. 23, 1701208 (2013).

${ }^{17}$ S. V. Bakurskiy, N. V. Klenov, I. I. Soloviev, V. V. Bol'ginov, V. V. Ryazanov, I. I. Vernik, O. A. Mukhanov, M. Y. Kupriyanov, and A. A. Golubov, Appl. Phys. Lett. 102, 192603 (2013).

${ }^{18}$ R. Caruso, D. Massarotti, A. Miano, V. V. Bol'ginov, A. Ben Hamida, L. N. Karelina, G. Campagnano, I. V. Vernik, F. Tafuri, V. V. Ryazanov, O. A. Mukhanov, and G. P. Pepe, IEEE Trans. Appl. Supercond. 28(7), 1800606 (2018).

${ }^{19}$ R. Caruso, D. Massarotti, V. V. Bolginov, A. Ben Hamida, L. N. Karelina, A. Miano, I. V. Vernik, F. Tafuri, V. V. Ryazanov, O. A. Mukhanov, and G. P. Pepe, J. Appl. Phys. 123, 133901 (2018).

${ }^{20}$ I. I. Soloviev, N. V. Klenov, S. V. Bakurskiy, V. V. Bol'ginov, V. V. Ryazanov, M. Yu Kupriyanov, and A. A. Golubov, Appl. Phys. Lett. 105, 242601 (2014).

${ }^{21}$ I. I. Soloviev, N. V. Klenov, S. V. Bakursky, M. Yu Kupriyanov, and A. A. Golubov, JETP Lett. 101, 240 (2015).

${ }^{22}$ S. V. Bakurskiy, N. V. Klenov, I. I. Soloviev, M. Yu Kupriyanov, and A. A. Golubov, Appl. Phys. Lett. 108, 042602 (2016).

${ }^{23}$ E. Goldobin, H. Sickinger, M. Weides, N. Ruppelt, H. Kohlstedt, R. Kleiner, and D. Koelle, Appl. Phys. Lett. 102(24), 242602 (2013).

${ }^{24}$ R. Menditto, H. Sickinger, M. Weides, H. Kohlstedt, M. Zonda, T. Novotny, D. Koelle, R. Kleiner, and E. Goldobin, Phys. Rev. B 93(17), 174506 (2016).

${ }^{25}$ J. J. A. Baselmans, T. T. Heikkila, B. J. van Wees, and T. M. Klapwijk, Phys. Rev. Lett. 89, 207002 (2002).
${ }^{26}$ S. M. Frolov, D. J. Van Harlingen, V. A. Oboznov, V. V. Bolginov, and V. V. Ryazanov, Phys. Rev. B 70, 144505 (2004).

${ }^{27}$ B. Baek, M. Schneider, M. R. Pufall, and W. H. Rippard, IEEE Trans. Appl. Supercond. 28(7), 1800705 (2018).

${ }^{28}$ M. J. A. Stoutimore, A. N. Rossolenko, V. V. Bolginov, V. A. Oboznov, A. Y. Rusanov, N. Pugach, S. M. Frolov, V. V. Ryazanov, and D. J. Van Harlingen, preprint arXiv:1805.12546 (2018).

${ }^{29}$ L. Trifunovic, Z. Popovic, and Z. Radovic, Phys. Rev. B 84, 064511 (2011).

${ }^{30}$ C. Richard, M. Houzet, and J. S. Meyer, Phys. Rev. Lett. 110(21), 217004 (2013).

${ }^{31}$ A. Buzdin, Phys. Rev. B 72, 100501(R) (2005).

${ }^{32}$ F. Konschelle, J. Cayssol, and A. I. Buzdin, Phys. Rev. B 78, 134505 (2008).

${ }^{33}$ A. Buzdin and A. E. Koshelev, Phys. Rev. B 67, 220504(R) (2003).

${ }^{34}$ N. G. Pugach, E. Goldobin, R. Kleiner, and D. Koelle, Phys. Rev. B 81(10), 104513 (2010).

${ }^{35}$ E. Goldobin, D. Koelle, R. Kleiner, and R. G. Mints, Phys. Rev. Lett. 107, 227001 (2011).

${ }^{36}$ S. V. Bakurskiy, N. V. Klenov, T. Yu. Karminskaya, M. Yu. Kupriyanov, and A. A. Golubov, Supercond. Sci. Technol. 26, 015005 (2013).

${ }^{37}$ H. Sickinger, A. Lipman, M. Weides, R. G. Mints, H. Kohlstedt, D. Koelle, R. Kleiner, and E. Goldobin, Phys. Rev. Lett. 109, 107002 (2012).

${ }^{38}$ S. V. Bakurskiy, N. V. Klenov, I. I. Soloviev, M. Y. Kupriyanov, and A. A. Golubov, Phys. Rev. B 88, 144519 (2013).

${ }^{39}$ I. P. Nevirkovets, O. Chernyashevskyy, G. V. Prokopenko, O. A. Mukhanov, and J. B. Ketterson, IEEE Trans. Appl. Supercond. 24(4), 1800506 (2014).

${ }^{40}$ N. Ruppelt, H. Sickinger, R. Menditto, E. Goldobin, D. Koelle, R. Kleiner, O. Vavra, and H. Kohlstedt, Appl. Phys. Lett. 106, 022602 (2015).

${ }^{41}$ S. Shafranjuk, I. P. Nevirkovets, O. A. Mukhanov, and J. B. Ketterson, Phys. Rev. Appl. 6, 024018 (2016).

${ }^{42}$ S. V. Bakurskiy, V. I. Filippov, V. I. Ruzhickiy, N. V. Klenov, I. I. Soloviev, M. Yu Kupriyanov, and A. A. Golubov, Phys. Rev. B 95, 094522 (2017).

${ }^{43}$ K. D. Usadel, Phys. Rev. Lett. 25, 507 (1970).

${ }^{44}$ M. Y. Kuprianov and V. F. Lukichev, Zh. Eksp. Teor. Fiz. 94, 139 (1988) [Sov. Phys. JETP 67, 1163 (1988)].

${ }^{45}$ D. M. Heim, N. G. Pugach, M. Yu, Kupriyanov, E. Goldobin, D. Koelle, R. Kleiner, N. Ruppelt, M. Weides, and H. Kohlstedt, New J. Phys. 17, 113022 (2015).

${ }^{46}$ N. Pompeo, K. Torokhtii, C. Cirillo, A. V. Samokhvalov, E. A. Ilyina, C. Attanasio, A. I. Buzdin, and E. Silva, Phys. Rev. B 90, 064510 (2014).

${ }^{47}$ A. V. Samokhvalov and A. I. Buzdin, Phys. Rev. B 92, 054511 (2015).

${ }^{48}$ S. N. Vdovichev, Y. N. Nozdrin, E. E. Pestov, P. A. Yunin, and A. V. Samokhvalov, JETP Lett. 104, 329 (2016).

${ }^{49}$ T. Golod, A. Iovan, and V. M. Krasnov, Nat. Commun. 6, 8628 (2015).

${ }^{50}$ A. Murphy, D. Averin, and A. Bezryadin, New J. Phys. 19(6), 063015 (2017). 